\title{
Corrigendum: Reviewing the Role of the Efferent Vestibular System in Motor and Vestibular Circuits
}

\author{
Miranda A. Mathews ${ }^{1}$, Aaron J. Camp ${ }^{1 *}$ and Andrew J. Murray ${ }^{2}$ \\ ${ }^{1}$ Sensory Systems and Integration Laboratory, Bosch Institute, Discipline of Biomedical Science, University of Sydney, \\ Sydney, NSW, Australia, ${ }^{2}$ Sainsbury Wellcome Centre for Neural Circuits and Behaviour, University College London, London, \\ United Kingdom
}

Keywords: efferent vestibular system, efferent vestibular nucleus, EVS, EVN, corollary discharge, VOR, vestibular, vestibular plasticity

\section{A corrigendum on}

Reviewing the Role of the Efferent Vestibular System in Motor and Vestibular Circuits by Mathews, M. A., Camp, A. J., and Murray, A. J. (2017). Front. Physiol. 8:552. doi: $10.3389 /$ fphys.2017.00552

In our original review article, there was a mistake in the reporting of Lysakowski and Singer (2000) in text and the placement of that publication in Figure 1. The original text on page 2 included the following statement:

"Studies in chinchilla present a variable picture from a single EVN cluster (Lysakowski and Singer, 2000) to three anatomically distinct groups near the facial nerve, abducens nerve, and vestibular nuclei (Marco et al., 1993).”

has been modified to:

"In other mammalian studies, more than one cluster was observed with the major nucleus being referred to as group $e$ (Goldberg and Fernàndez, 1980), located dorsal and/or ventral to the facial nerve (Shumilina et al., 1986; Perachio and Kevetter, 1989). Smaller clusters are scattered in the caudal pontine reticular nucleus and the medial reticular nucleus (Strutz, 1982a,b). Interestingly, in the chinchilla, three anatomically distinct groups near the facial nerve, abducens nerve, and vestibular nuclei were distinguished (Marco et al., 1993; Lysakowski and Singer, 2000), though the cluster ventral to the facial nerve likely reflects projections to the middle ear rather than the peripheral vestibular labyrinth (Lysakowski and Singer, 2000)."

Figure 1 has also been amended in line with this modification, as well as a typographical correction of "squirril monkey" to "squirrel monkey" under (Goldberg and Fernàndez, 1980) in the "Three Clusters" section. The figure legend has also been amended to clarify these changes. The amended Figure 1 is now:

Figure 1 legend has been modified from:

"Figure 1. Anatomy and morphology of the EVS across vertebrates. Studies that directly investigated EVS anatomy and morphology were separated under the following categories-cell body clustering, innervation pattern, and dendritic arborization. Studies that assessed more than one category are mentioned in each respective category they investigated. Where more than one cell body cluster was observed, the number of clusters is labeled and depicted with the respective number of blue pictorial clusters. Uni- and bi-lateral projections are also labeled and depicted with pink lines from a coronal brainstem schematic out toward the inner ear (drawings not to scale). Only one bilateral projection is drawn for Meredith and Roberts (1987) eel as they denoted it as a minor finding. Expansive green lines along the brainstem tegmentum denote 


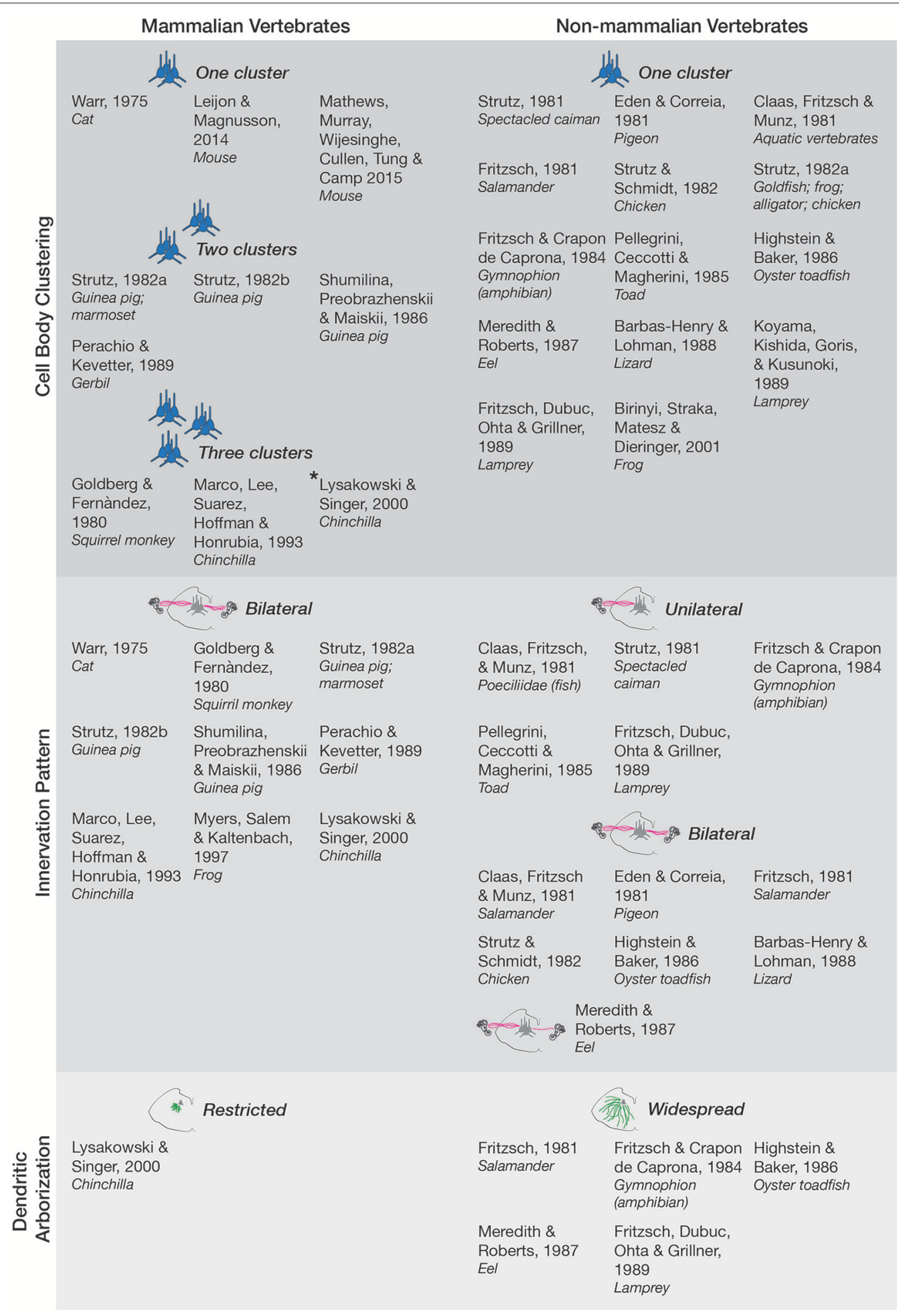

FIGURE 1 | Anatomy and morphology of the EVS across vertebrates. Studies that directly investigated EVS anatomy and morphology were separated under the following categories-cell body clustering, innervation pattern, and dendritic arborization. Studies that assessed more than one category are mentioned in each respective category they investigated. Where more than one cell body cluster was observed, the number of clusters is labeled and depicted with the respective number of blue pictorial clusters. Asterisk next to Lysakowski and Singer (2000) denotes one cluster likely projecting to middle ear instead of peripheral vestibular labyrinth. Uni- and bilateral projections are also labeled and depicted with pink lines from a coronal brainstem schematic out towards the inner ear (drawings not to scale). Only one bilateral projection is drawn for Meredith and Roberts (1987) eel as they denoted it as a minor finding. Expansive green lines along the brainstem tegmentum denote widespread arborization of dendrites, and shorter green lines depict restricted arborization, as labeled. Nonmammalian species included all animals groups not classified as mammals. 
widespread arborization of dendrites, and shorter green lines depict restricted arborization, as labeled. Non-mammalian species included all animals groups not classified as mammals."

to:

"Figure 1. Anatomy and morphology of the EVS across vertebrates. Studies that directly investigated EVS anatomy and morphology were separated under the following categories-cell body clustering, innervation pattern, and dendritic arborization. Studies that assessed more than one category are mentioned in each respective category they investigated. Where more than one cell body cluster was observed, the number of clusters is labeled and depicted with the respective number of blue pictorial clusters. Asterisk next to Lysakowski and Singer (2000) denotes

\section{REFERENCES}

Goldberg, J. M., and Fernàndez, C. (1980). Efferent vestibular system in the squirrel monkey: anatomical location and influence on afferent activity. J. Neurophysiol. 43, 986-1025.

Lysakowski, A., and Singer, M. (2000). Nitric oxide synthase localized in a subpopulation of vestibular efferents with NADPH diaphorase histochemistry and nitric oxide synthase immunohistochemistry. J. Comp. Neurol. 427, 508-521. doi: 10.1111/j.1749-6632.1996. tb15752.x

Marco, J., Lee, W., Suarez, C., Hoffman, L., and Honrubia, V. (1993). Morphologic and quantitative study of the efferent vestibular system in the chinchilla: 3-D reconstruction. Acta Otolaryngol. 113, 229-234. doi: $10.3109 / 00016489309135798$

Meredith, G. E., and Roberts, B. L. (1987). Distribution and morphological characteristics of efferent neurons innervating end organs in the ear and lateral line of the European eel. J. Comp. Neurol. 265, 494-506. doi: $10.1002 / \mathrm{cne} .902650404$

Perachio, A. A., and Kevetter, G. A. (1989). Identification of vestibular efferent neurons in the gerbil: histochemical and retrograde labelling. Exp. Brain Res. 78, 315-326. doi: 10.1007/BF00228903 one cluster likely projecting to middle ear instead of peripheral vestibular labyrinth. Uni- and bi-lateral projections are also labeled and depicted with pink lines from a coronal brainstem schematic out towards the inner ear (drawings not to scale). Only one bilateral projection is drawn for Meredith and Roberts (1987) eel as they denoted it as a minor finding. Expansive green lines along the brainstem tegmentum denote widespread arborization of dendrites, and shorter green lines depict restricted arborization, as labeled. Non-mammalian species included all animals groups not classified as mammals."

The authors sincerely apologize for the ambiguities. These changes do not significantly alter the review article.

The original article has been updated.

Shumilina, V. F., Preobrazhenskii, N. N., and Maiskii, V. A. (1986). Study of vestibular efferent neurons of the guinea pig by the technic of retrograde axonal transport of horseradish peroxidase and with fluorochromes. Neirofiziologiia $18,738-747$

Strutz, J. (1982a). The origin of efferent labyrinthine fibres: a comparative study in vertebrates. Arch. Otorhinolaryngol. 234, 139-143. doi: 10.1007/BF00453620

Strutz, J. (1982b). The origin of efferent vestibular fibres in the guinea pig. A horseradish peroxidase study. Acta Otolaryngol. 94, 299-305. doi: $10.3109 / 00016488209128917$

Conflict of Interest Statement: The authors declare that the research was conducted in the absence of any commercial or financial relationships that could be construed as a potential conflict of interest.

Copyright (c) 2018 Mathews, Camp and Murray. This is an open-access article distributed under the terms of the Creative Commons Attribution License (CC BY). The use, distribution or reproduction in other forums is permitted, provided the original author(s) and the copyright owner are credited and that the original publication in this journal is cited, in accordance with accepted academic practice. No use, distribution or reproduction is permitted which does not comply with these terms. 